Europhysics Letters

PREPRINT

\title{
Aging dynamics and density relaxation in kinetic lattice gases under gravity
}

\author{
Y. $\operatorname{Levin}^{1}(*)$, J. J. Arenzon ${ }^{1}(* *)$ and M. $\operatorname{Sellitto~}^{2}(* *)$ \\ 1 Instituto de Física, Universidade Federal do Rio Grande do Sul \\ Caixa Postal 15051, CEP 91501-970, Porto Alegre, RS, Brazil \\ 2 Laboratoire de Physique, École Normale Supérieure de Lyon - 46 Allée d'Italie, F- \\ 69364 Lyon cedex 07, France
}

PACS. 05.40.-a - ...

PACS. 05.70.Fh $-\ldots$.

\begin{abstract}
We present an analytical approach to the out of equilibrium dynamics of a class of kinetic lattice gases under gravity. The location of the jamming transition, the critical exponents, and the scaling functions characterizing the relaxation processes are determined. In particular, we find that logarithmic compaction and simple aging are intimately related to the Vogel-Fulcher law, while power-law compaction and super-aging behavior occur in presence of a power-law diffusion.
\end{abstract}

Granular materials set in rapid motion by vibration [1] exhibit features such as universal velocity distribution [2], and "de Gennes narrowing" - a physical signature of the cage effect [3] - which in spite of the non-equilibrium nature of the stationary state, closely resemble those observed in simple liquids at thermal equilibrium 肺. In the opposite, quasistatic flow limit, slow compaction phenomena appear [5]. During compaction, the free volume available to grains decreases, and the mobility steeply falls to zero, hence aging phenomena are expected to occur [6 8], as is confirmed in several numerical simulations [9 [12]. It has been suggested that in this regime a granular material should resemble a highly viscous liquid or a glass 13, and several approaches have been proposed to describe different aspects of the granular compaction dynamics. These are mainly based on Langevin 14] and Fokker-Planck equation [15, fluctuating nonlinear hydrodynamics [16, and mode-coupling theory 17.

Two effects are responsible for the unusual behavior of a compacting granular material. First, collisions between the particles are inelastic, and energy has to be constantly pumped into the system. Second, at high packing density, steric hindrance, and the associated cage effect, play a crucial role very similar to the one observed in amorphous systems. In this letter we shall be concerned precisely with this second effect, which in the case of gentle shaking is the dominant one. A number of lattice-gas models have been introduced to study numerically the slow dynamics induced by this effect 18 20]. Our approach allows to characterize in a

$\left({ }^{*}\right)$ E-mail: levin@if.ufrgs.br

$(* *)$ E-mail: arenzon@if.ufrgs.br

$(* * *)$ E-mail: msellitt@ens-lyon.fr

(C) EDP Sciences 
precise way the density relaxation and the aging dynamics in terms of the particle mobility. We show the existence of two relaxation regimes (exponential and aging) separated by a jamming transition. Using scaling arguments we investigate the asymptotic long-time behavior of the density and of the mean-square displacement, and compare the results with the numerical solution of the diffusion equation. We also check our analytic results with the Monte Carlo simulation of a gravity-driven version of the Kob-Andersen model (KA) [18, 21]. In this model, non-interacting particles are allowed to move only if a local kinetic constraint on the occupation of the nearest neighboring sites is satisfied.

The model. - Consider a box of height $H$ in contact with a particle reservoir of density $\rho_{\mathrm{R}}$ located at $z=H$. All lengths are measured in units of the particle diameter. A constant gravitational field of strength $g$ acts in the $-z$ direction, forcing particles from the reservoir to enter into the box. The dynamical evolution of the local particle density $\rho(z, t)$ is governed by the continuity equation, $\partial_{t} \rho(z, t)+\partial_{z} J(z, t)=0$, with current given by the Fick's law, $J(z, t)=-\Gamma(\rho) \partial_{z} \mu(z, t) . \Gamma(\rho)$ is the Onsager mobility and $\mu(z, t)=\frac{\delta F}{\delta \rho}$ is the local chemical potential. For simplicity we shall suppose that the only interaction between the grains is due to the hard core repulsion. The exact Helmholtz free energy functional [23] for the lattice gas version of this model is

$$
F[\rho(z, t)]=k_{\mathrm{B}} T \int_{0}^{H} d z[(1-\rho) \ln (1-\rho)+\rho \ln \rho+\gamma z \rho],
$$

where $\gamma=m g / k_{\mathrm{B}} T$ is the inverse gravitational length. In the case of granular materials the thermal energy of the grains is negligible so that $T$ is a function of the externally imposed vibration intensity [27]. It is neither the physical temperature nor the "granular temperature" usually associated with the average kinetic energy [1]. For the KA model, the mobility $\Gamma(\rho)$ vanishes as $\Gamma(\rho)=\Gamma_{0} \rho\left(1-\rho / \rho_{\mathrm{c}}\right)^{\phi}$, with the critical threshold $\rho_{\mathrm{c}}=0.88$ for the simple cubic lattice [18], and $\rho_{c}=0.81$ for the BCC lattice. In both cases $\phi=3.1$. A similar expression is found for the mobility of highly packed hard-sphere systems [24, 25]. One should note, however, that the free energy functional given by Eq. (11) is exact only for the lattice gas. In the case of hard spheres it can be replaced by an approximate expression derived from the Carnahan-Starling equation of state [23, 26], without any qualitative modifications to the theory. Substituting $\Gamma(\rho)$ into the continuity equation we are lead to,

$$
\frac{\partial \rho(z, t)}{\partial t}=\frac{\partial}{\partial z}\left\{\left(1-\frac{\rho}{\rho_{\mathrm{c}}}\right)^{\phi}\left[\frac{1}{1-\rho} \frac{\partial \rho}{\partial z}+\gamma \rho\right]\right\},
$$

where the time is now measured in units of $1 / \Gamma_{0} k_{\mathrm{B}} T$. We should stress that the local density approximation in which the functional form of mobility is assumed to remain valid in an inhomogeneous system is highly non-trivial. One of the goals of this paper is to assess to what extent this approximation works in the case of kinetic lattice-gas models.

The boundary conditions require vanishing of the current at $z=0, J(0, t)=0$; and $\rho(H, t)=\rho_{\mathrm{R}}$. The stationary state is obtained when $\partial \rho / \partial t=0$, which implies that $J(z, \infty)=0$ for all $z$. The jamming transition corresponds to the locus in the parameter space $\left(\gamma, \rho_{\mathrm{R}}\right)$, at which a layer of critical density first appears, $\rho(0, \infty)=\rho_{c}$. This happens when

$$
\gamma_{\mathrm{c}}\left(\rho_{\mathrm{R}}\right)=\frac{1}{H} \ln \left[\frac{\rho_{\mathrm{c}}\left(1-\rho_{\mathrm{R}}\right)}{\rho_{\mathrm{R}}\left(1-\rho_{\mathrm{c}}\right)}\right] .
$$

Depending on the value of $\gamma$ two very different types of stationary profiles are found. In Fig. 囵 we compare these with the stationary profiles found from the Monte Carlo simulation of the 
gravity-driven KA model on the BCC lattice [21]. A perfect agreement is obtained with no adjustable parameters. It is important to note that for $\gamma>\gamma_{\mathrm{c}}\left(\rho_{\mathrm{R}}\right)$ the stationary profiles are no longer equilibrium distributions, i.e. they do not minimize the Helmholtz free energy functional Eq. 11.

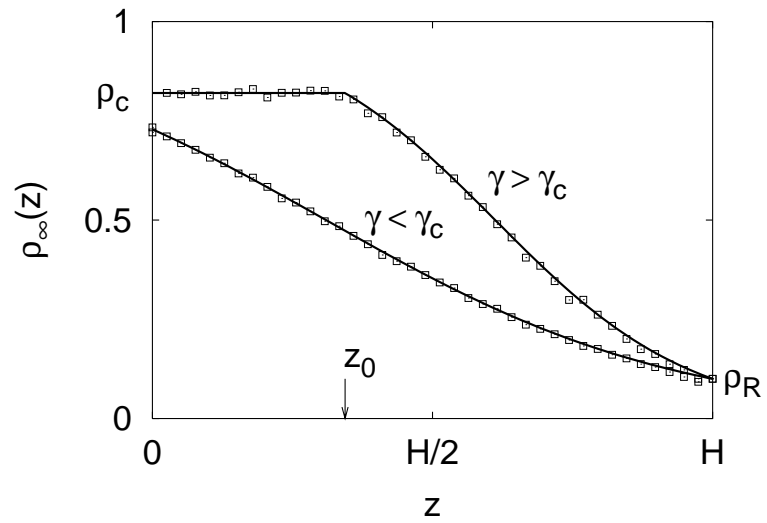

Fig. 1 - Examples of the stationary profiles above and below the critical temperature (solid lines). For $\gamma \leq \gamma_{\mathrm{c}}\left(\rho_{\mathrm{R}}\right)$ (high temperature) the equilibrium density profile is $\rho_{\infty}(z)=1 /[\exp (\gamma z+\eta)+1]$, with $\eta=\ln \left[\left(1-\rho_{\mathrm{R}}\right) / \rho_{\mathrm{R}}\right]-\gamma H$. At low temperatures, $\gamma>\gamma_{\mathrm{c}}\left(\rho_{\mathrm{R}}\right)$, the stationary profile is $\rho_{\infty}(z)=\rho_{\mathrm{c}}$ for $z \leq z_{0}\left(\rho_{\mathrm{R}}\right) \equiv H\left[1-\gamma_{\mathrm{c}}\left(\rho_{\mathrm{R}}\right) / \gamma\right]$; and $\rho_{\infty}(z)=1 /[\exp (\gamma z+\eta)+1]$ for $z>z_{0}\left(\rho_{\mathrm{R}}\right)$. The squares are the asymptotic densities obtained through simulation of the gravity-driven KA model on the BCC lattice (see 21] for details on simulation).

High-temperature phase, $\gamma<\gamma_{\mathrm{c}}\left(\rho_{\mathrm{R}}\right)$. - At high temperature we expect an exponential relaxation towards the equilibrium distribution, $\rho(z, t) \asymp \rho_{\infty}(z)+g(z) \mathrm{e}^{-t / \tau}$. We have solved Eq. (2) numerically and checked that the approach to equilibrium is indeed exponentially fast for any $\gamma<\gamma_{\mathrm{c}}\left(\rho_{\mathrm{R}}\right)$. To see how the characteristic time $\tau$ depends on the various parameters of the system we perform a scaling analysis of Eq. (2). We first note that associated with the two right-hand terms of Eq. (2) there are two temporal scales. These can be identified as the diffusion time $\tau_{1} \propto H^{2}$, and the drift time $\tau_{2} \propto H / \gamma$. The characteristic time must, therefore, satisfy an exact equation $\tau^{-1}=\frac{\pi^{2}}{4 H^{2}} \mathcal{F}\left(\gamma H ; \rho_{\mathrm{R}}\right)$, where $\mathcal{F}\left(x ; \rho_{\mathrm{R}}\right)$ is a scaling function. The reason for the prefactor $\pi^{2} / 4$ will become clear from the forthcoming analysis. To further explore the properties of Eq. (2) we now study its linear version,

$$
\frac{\partial \rho(z, t)}{\partial t}=\frac{\partial^{2} \rho}{\partial z^{2}}+\gamma \frac{\partial \rho}{\partial z}
$$

For Eq. (4), the characteristic time of approach to equilibrium can be related to the singularities of the temporal Laplace transform of the density profile. For $\tau>4 / \gamma^{2}$ the characteristic time is found to satisfy

$$
\exp \left\{H \sqrt{\gamma^{2}-4 \tau^{-1}}\right\}=\frac{\gamma+\sqrt{\gamma^{2}-4 \tau^{-1}}}{\gamma-\sqrt{\gamma^{2}-4 \tau^{-1}}}
$$

while for $\tau<4 / \gamma^{2}$ it satisfies

$$
\cos \left\{H \sqrt{4 \tau^{-1}-\gamma^{2}}\right\}=\tau \gamma^{2} / 2-1
$$


We note that for $\gamma=0, \tau^{-1}=\pi^{2} / 4 H^{2}$. For finite $\gamma$ the inverse relaxation time for linear Eq. (4) can be written in a scaling form, $\tau^{-1}=\frac{\pi^{2}}{4 H^{2}} f(\gamma H)$. Unlike the scaling function $\mathcal{F}\left(x ; \rho_{\mathrm{R}}\right)$ for the non-linear Eq. (2), $f(x)$ does not depend on the reservoir density. For $x=0$, $f(0)=1$; for large $x, f(x) \asymp\left(4 / \pi^{2}\right) x^{2} \exp (-x)$. The graphs of $f(x)$ and of its asymptotic form are presented in Fig. 2.

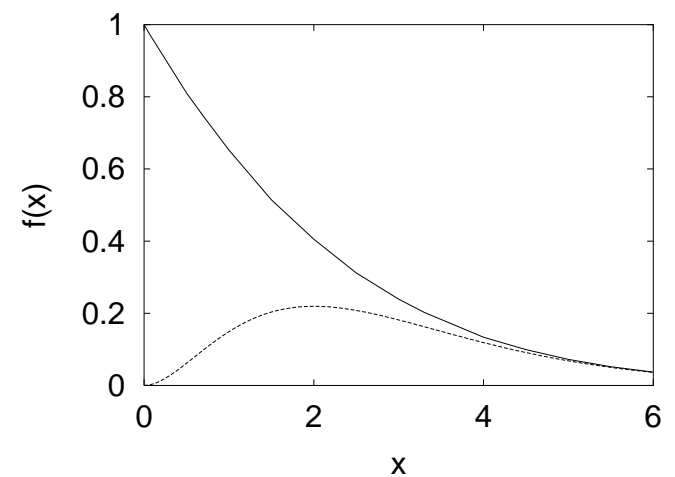

Fig. 2

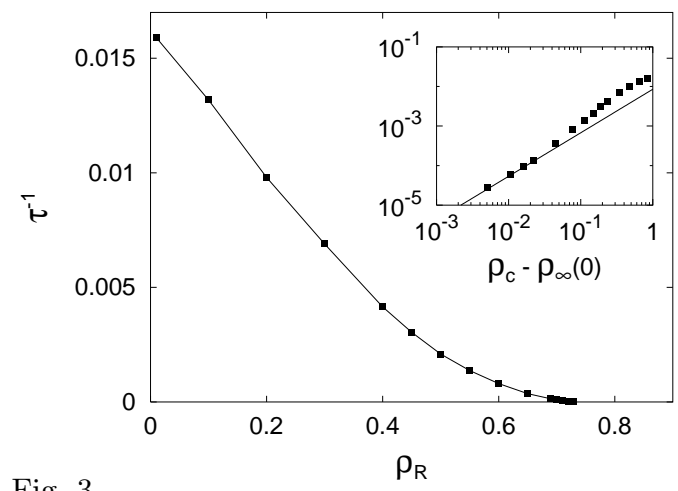

Fig. 3

Fig. 2 - Scaling function $f(x)$ (solid curve) and its asymptotic form (dashed line) as discussed in text.

Fig. 3 - Inverse relaxation time, $\tau^{-1}$, for $\gamma=0.1$ and $H=10$ with $\rho_{c}=0.88$ and $\phi=3.1$, corresponding to the Kob-Andersen model on a simple cubic lattice. The points are the result of numerical integration of Eq. (2). Inset shows the same data on the log scale. The characteristic time diverges with exponent $\phi-2$ as the jamming transition is approached.

With the insight gained from the study of Eq. (4) we can now explore the full non-linear Eq. (2). We first consider the special case of zero gravity 28]. For $\gamma=0$ particles diffuse freely from the reservoir until a uniform density profile $\rho_{\infty}(z)=\rho_{\mathrm{R}}$ is established. At a low reservoir density $\rho_{\mathrm{R}} \ll \rho_{\mathrm{c}}$, we expect that the non-linearities of Eq. (2) should be irrelevant and the relaxation time should reduce to $\tau^{-1} \approx \frac{\pi^{2}}{4 H^{2}}$. Linearizing Eq.(2) around the equilibrium state we find that the relaxation time for $\gamma=0$ is

$$
\tau^{-1}=\frac{\pi^{2}}{4 H^{2}\left(1-\rho_{\mathrm{R}}\right)}\left(1-\frac{\rho_{\mathrm{R}}}{\rho_{\mathrm{c}}}\right)^{\phi},
$$

or equivalently $\mathcal{F}\left(0 ; \rho_{\mathrm{R}}\right)=\left(1-\rho_{\mathrm{R}} / \rho_{\mathrm{C}}\right)^{\phi} /\left(1-\rho_{\mathrm{R}}\right)$. Eq. (7) is in perfect agreement with the numerical integration of Eq. (2). As expected, the relaxation time diverges as $\rho_{\mathrm{R}} \rightarrow \rho_{\mathrm{C}}$. The exponent characterizing this divergence is $\phi$.

Finally in the presence of a gravitational field we find, by numerical integration of Eq.(2), that as $\gamma \rightarrow \gamma_{\mathrm{c}}\left(\rho_{\mathrm{R}}\right)$, the density of the first layer approaches $\rho_{c}, \rho_{\infty}(0) \rightarrow \rho_{c}$, and $\mathcal{F} \sim$ $\left(\gamma_{c}-\gamma\right)^{\phi-2}$. The relaxation time diverges with exponent $\phi-2$, see Fig 3 , implying that the dynamics is faster than in the zero gravity case. Comparing with eq. (7), we see that the jamming transitions in the homogeneous and inhomogeneous systems, therefore, belong to distinct universality classes.

Low-temperature dynamics, $\gamma>\gamma_{\mathrm{c}}\left(\rho_{\mathrm{R}}\right)$. - At low temperatures, the density of the lower layers closely approaches the critical threshold, $\rho_{c}$. At such large packing fractions the movement of particles is highly restricted, and even the slightest increase of density requires a rearrangement of a huge number of grains. In this regime we expect slow relaxation and aging 
phenomena to appear. To check this supposition one should solve Eq. (2). Unfortunately, due to its highly non-linear nature no analytic solution is possible. Nevertheless an asymptotic solution can be found. Let us focus our attention on the bottom layers, $z<z_{0}\left(\rho_{\mathrm{R}}\right)$, of the

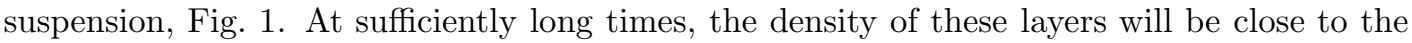
critical one, $\rho(z, t) \simeq \rho_{\mathrm{c}}$. To the lowest order in $\Delta(z, t) \equiv 1-\rho(z, t) / \rho_{\mathrm{c}}$, Eq. (2) simplifies to,

$$
\frac{\partial \Delta(z, t)}{\partial t}=-\gamma \frac{\partial \Delta^{\phi}}{\partial z}
$$

To solve this non-linear equation we propose a scaling ansatz $\Delta(z, t)=\Delta\left(z / t^{\alpha}\right)$. Substituting into Eq. (8) we see that this form is a solution if $\alpha=1$ and

$$
\Delta(z, t)=\left[\frac{z}{\phi \gamma t}\right]^{\frac{1}{\phi-1}} .
$$

To check the asymptotic solution, Eq. (9), we have numerically integrated Eq. (2) for $\gamma>$ $\gamma_{\mathrm{c}}\left(\rho_{\mathrm{R}}\right)$. For large times a perfect agreement between the numerical solution and the Eq. (9) is found, as can be seen from Fig. 4. In the absence of gravity, $\gamma=0$, relaxation is slower and characterized by a different dynamic exponent, $\Delta(z, t) \sim t^{-1 / \phi}$ [28]. The same behavior is observed in the KA model (see ref. 28] and Fig. 5).

Up to now our discussion has been motivated by the dynamics of the KA model which is characterized by a power law mobility coefficient. However, there are various systems for which the mobility vanishes according to the Vogel-Fulcher law, $\Gamma(\rho)=\Gamma_{0} \rho \exp \left[a \rho_{\mathrm{c}} /\left(\rho-\rho_{\mathrm{c}}\right)\right]$. In this case an analysis similar to the one presented above leads to an asymptotic scaling solution, which for very large times reduces to

$$
\Delta(z, t)=\frac{a}{\ln (t / z)}
$$

Interestingly, a similar logarithmic law was discovered in the granular compaction experiments [29], and is supported by several analytical approaches 30 34.

We now turn to the discussion of the aging phenomena [7, 8, 35, 36]. Consider the two-times mean square displacement of grains, $B\left(t, t_{\mathrm{w}}\right)$. If $t$ is sufficiently larger than $t_{\mathrm{w}}$, this can be written as

$$
B\left(t, t_{\mathrm{w}}\right)=\int_{t_{\mathrm{w}}}^{t} d s \Gamma[\rho(z, s)] .
$$

For power-law diffusion and $z<z_{0}\left(\rho_{\mathrm{R}}\right)$ we find, to leading order in $t$ and $t_{\mathrm{w}}$,

$$
B\left(t, t_{\mathrm{w}}\right) \sim t_{\mathrm{w}}^{1-\mu}-t^{1-\mu}
$$

with the exponent $\mu=\phi /(\phi-1)$. Usually $\phi>1$, so that $\mu>1$, which corresponds to a super-aging regime [7]. This means that the effective structural relaxation time grows as $t_{\mathrm{w}}^{\mu}$, what is faster than the age of the system, $t_{\mathrm{w}}$ [7]. This scaling behavior has been observed in the simulation of the gravity-driven KA model [39. The comparison with the zero-gravity case, for which a simple aging $\left(t / t_{\mathrm{w}}\right)$ was found [28], once again shows the important role played by gravity.

For the Vogel-Fulcher law we find, to the leading order in $t$ and $t_{\mathrm{w}}$ (and $\left.z<z_{0}\left(\rho_{\mathrm{R}}\right)\right)$, a simple aging scenario, $B\left(t, t_{\mathrm{w}}\right) \sim \log \left(t / t_{\mathrm{w}}\right)$. It is interesting to observe the different behavior of this function and (12) at finite waiting times $t_{\mathrm{w}}$. In the former case, $\lim _{t \rightarrow \infty} B\left(t, t_{\mathrm{w}}\right)=\infty$, i.e., a weak ergodicity scenario [37]; while in the latter, a finite limit is obtained (which, 


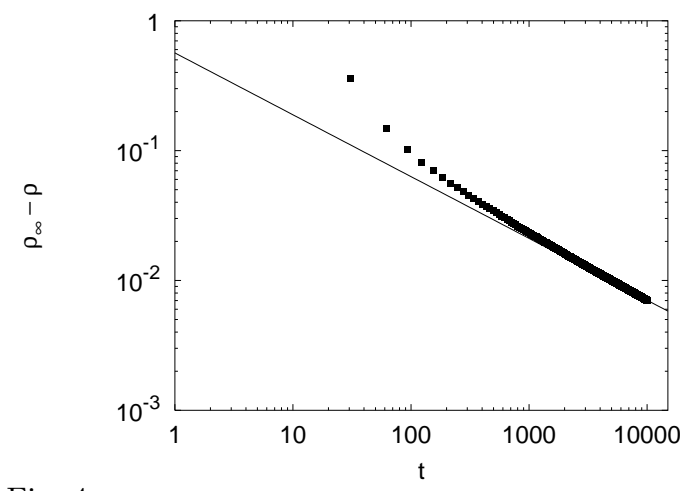

Fig. 4

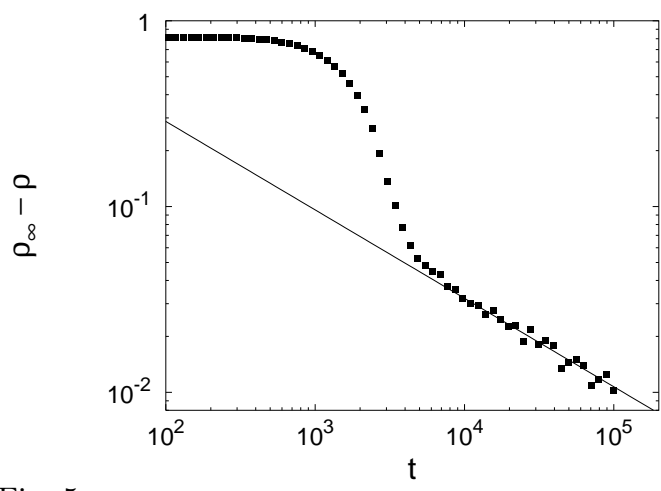

Fig. 5

Fig. 4 - Power-law density relaxation for $\gamma>\gamma_{\mathrm{c}}\left(\rho_{\mathrm{R}}\right)$. The points are result of numerical integration of the Eq. (8), while the solid line is the asymptotic solution given by Eq. (9).

Fig. 5 - Density relaxation found in the simulation of the gravity driven KA model on the BCC lattice. Averages are over 100 samples and $H=4 L=80, \rho_{\mathrm{R}}=0.1$, and $\gamma=0.1$. The line is the theoretical prediction $t^{-1 /(\phi-1)}$.

however, vanishes as $\left.t_{\mathrm{w}} \rightarrow \infty\right)$. Nevertheless, the manner in which time-translation invariance is violated is, in a sense, similar. Indeed, if we consider the "triangle relation" 38], $B\left(t_{1}, t_{3}\right)=f\left[B\left(t_{1}, t_{2}\right), B\left(t_{2}, t_{3}\right)\right]$, where the times $t_{1}, t_{2}$, and $t_{3}$ are in increasing order, it is straightforward to check that in both Vogel-Fulcher and power law cases $f(x, y)=x+y$, implying that displacements over non-overlapping time intervals are statistically independent. This feature does not hold in the presence of activated aging for which $B\left(t, t_{\mathrm{w}}\right) \sim \log t / \log t_{\mathrm{w}} 8$.

Conclusions. - To summarize, we have presented an analytical study of the dynamics of a class of kinetic lattice models. Our approach based on the local density approximation for the Onsager mobility allows us to predict the density relaxation law and the nature of aging behavior. The results reproduce successfully the behavior observed in the numerical simulation of the KA and gravity-driven KA model, and they could be relevant to systems for which steric hindrance and cage effect are dominant, such as polydispersed colloidal suspension in gravitational field [24, 25], and slowly compacting dense granular matter. For hard-sphere systems it is known that the diffusion coefficient vanishes as a power-law with $\phi$ between 2 and 3 , and the sedimentation profile is approached very slowly (one year, roughly) [40]; hence a super-aging behavior in the two-times mean-square height displacement with exponent $\mu$ between $3 / 2$ and 2 should be observed.

$$
* * *
$$

We thank J. Kurchan and D. Stariolo for discussions and a critical reading of the manuscript. We are grateful to the referee for noticing an error in Eq. (7) of the original manuscript. This work was supported in part by the Brazilian agencies CNPq and FAPERGS. MS is supported by a Marie Curie fellowship of the EU (contract ERBFMBICT983561).

\section{REFERENCES}

[1] C.S. Campbell Annu. Rev. Fluid Mech. 2257 (1990). 
[2] F. Rouyer and N. Menon Phys. Rev. Lett. 853676 (2000).

[3] S. Warr and J.-P. Hansen Europhys. Lett. 36589 (1996).

[4] J.-P. Hansen and I.R. McDonald Theory of Simple Liquids (Academic, London, 1986).

[5] H.M. Jaeger, S.R. Nagel, and R.P. Behringer, Rev. Mod. Phys 68, 1259 (1996).

[6] L.C.E. Struik, Physical Aging in Amorphous Polymers and Other materials, (Elsevier, Houston, 1978), Chap. 7.

[7] J.-P. Bouchaud, Aging in glassy systems: new experiments, simple models, and open questions, in ref. [22].

[8] J.-P. Bouchaud, L.F. Cugliandolo, J. Kurchan and M. Mézard, in Spin-glasses and random fields, A.P. Young ed. (World Scientific, Singapore, 1997).

[9] M. Nicodemi and A. Coniglio Phys. Rev. Lett. 82916 (1999);

[10] A. Barrat and V. Loreto J. Phys. A 334401 (2000);

[11] J. Talbot, G. Tarjus and P. Viot cond-mat/0008183

[12] P. Philippe and D. Bideau, Phys. Rev. E 63051304 (2001).

[13] S.F. Edwards, in: Granular Matter: An Interdisciplinary Approach, A. Mehta, Ed. (SpringerVerlag, New York, 1994).

[14] A. Mehta, R.J. Needs, S. Dattagupta, J. Stat. Phys. 68, 1131 (1992).

[15] S.F. Edwards and D.V. Grinev Phys. Rev. E 584578 (1998).

[16] S.A. Hill and G.F. Mazenko Phys. Rev. E 63031303 (2001).

[17] L. Berthier, L.F. Cugliandolo, and J.L. Iguain Phys. Rev. E 63051302 (2001).

[18] W. Kob and H.C. Andersen Phys. Rev. E 484364 (1993).

[19] E. Caglioti, V. Loreto, H.J. Herrmann and M. Nicodemi, Phys. Rev. Lett. 791575 (1997).

[20] A. Mehta and G.C. Barker, J. Phys. C 126619 (2000).

[21] M. Sellitto and J.J. Arenzon Phys. Rev. E 627793 (2000).

[22] Soft and Fragile Matter: Non-equilibrium Dynamics, Metastability and Flow edited by M.E. Cates and M.R. Evans (IoP, 2000)

[23] Y. Levin Physica A 287100 (2000).

[24] W. Götze and L. Sjögren, Rep. Prog. Phys. 55241 (1992).

[25] M. Tokuyama and I. Oppenheim, Physica A 21685 (1995).

[26] N.F. Carnahan and K.E. Starling J. Chem. Phys. 51635 (1969).

[27] H. Hayakawa and D.C. Hong Phys. Rev. Lett. 782764 (1997); Inter. J. Bifurcation and Chaos 71159 (1997).

[28] L. Peliti and M. Sellitto, J. Phys. IV France 8 Pr6 49 (1998).

[29] J.B. Knight, C.G. Fandrich, C.N. Lau, H.M. Jaeger and S.R. Nagel, Phys. Rev. E 513957 (1995).

[30] T. Boutreux and P.-G. de Gennes Physica A 24459 (1997).

[31] E. Ben-Naim, J.B. Knight, E.R. Nowak, H.M. Jaeger and S.R. Nagel, Physica D 123380 (1998).

[32] J.J. Brey, A. Prados and B. Sánchez-Rey Phys. Rev. E 605685 (1999).

[33] D.A. Head Phys. Rev. E 62, 2439 (2000).

[34] S.N. Majumdar, D.S. Dean, and P. Grassberger Phys. Rev. Lett. 862301 (2001).

[35] D.A. Stariolo Phys. Rev. E 554806 (1997).

[36] L. Laloux, and P. Le Doussal Phys. Rev. E 576296 (1998).

[37] J.-P. Bouchaud J. Phys. I (France) 21705 (1992).

[38] L.F. Cugliandolo and J. Kurchan J. Phys. A 275749 (1994).

[39] M. Sellitto, Phys. Rev. E 63060301 (2001).

[40] P.M. Chaikin, Thermodynamics and hydrodynamics of hard sphere: the role of gravity, in ref. [22], and references therein. 